OPEN ACCESS

Edited by:

Hongji Dai,

Tianjin Medical University Cancer

Institute and Hospital, China

Reviewed by:

Yongfu Yu,

Aarhus University, Denmark

Agnieszka Micek,

Jagiellonian University, Poland

*Correspondence:

Jian Wang

wangjian@xinhuamed.com.cn

Sun Chen

chengsun@hotmail.com

Kun Sun

sunkun@xinhuamed.com.cn

${ }^{t}$ These authors have contributed equally to this work and share first authorship

FThese authors have contributed equally to this work

Specialty section:

This article was submitted to

Nutritional Epidemiology,

a section of the journal

Frontiers in Nutrition

Received: 10 August 2021 Accepted: 22 September 2021

Published: 26 October 2021

Citation:

Wang H, Du B, Wu Y, Li Z, Niu Y, Ouyang $F$, Wang J, Chen $S$ and Sun $K$ (2021) Sex-Disparity in the Association Between Birthweight and

Cardiovascular Parameters in

4-Year-Old Children: A Chinese Cohort Study. Front. Nutr. 8:756512.

doi: 10.3389/fnut.2021.756512

\section{Sex-Disparity in the Association Between Birthweight and Cardiovascular Parameters in 4-Year-Old Children: A Chinese Cohort Study}

\author{
Hualin Wang ${ }^{1 \dagger}$, Bowen Du ${ }^{1 \dagger}$, Yujian $\mathrm{Wu}^{2}$, Zhuoyan $\mathrm{Li}^{1}$, Yiwei Niu ${ }^{1}$, Fengxiu Ouyang ${ }^{3}$, \\ Jian Wang ${ }^{1 * \neq}$, Sun Chen ${ }^{1 * \neq}$ and Kun Sun ${ }^{1 * \neq}$ \\ 'Department of Pediatric Cardiology, Xinhua Hospital, School of Medicine, Shanghai Jiao Tong University, Shanghai, China, \\ ${ }^{2}$ Heart Center, Guangzhou Women and Children's Medical Center, Guangzhou, China, ${ }^{3}$ Ministry of Education, Shanghai Key \\ Laboratory of Children's Environmental Health, Xinhua Hospital, School of Medicine, Shanghai Jiao Tong University, \\ Shanghai, China
}

Background: Sex-related differences in cardiovascular parameters have been well documented in adults, and the impact of birthweight on cardiovascular health in later life has been acknowledged. However, data was limited regarding the association between birthweight and cardiovascular outcomes at an early age, and the sex-disparity in the association remained unclear.

Objective: To investigate the association between birthweight and cardiovascular parameters in 4-year-old children. Furthermore, to explore whether sex-disparity exist in this association or in cardiovascular risk.

Methods: Follow-up data from the Shanghai Birth Cohort (SBC) was analyzed. Detailed perinatal information including both maternal and offspring datum were recorded. Blood pressure, echocardiography, and anthropometry assessment were conducted during the follow-up of 4-year-old children. Linear regression models were used to analyze the association between birthweight and left ventricle (LV) structure and function changes in each sex and birthweight category. Multivariable logistic regression models were used to compare risk of left ventricular hypertrophy (LVH) in different birthweight subgroups.

Results: Overall, macrosomia was significantly associated with thickened LV posterior wall thickness in systole [LVPWs, $(\beta=0.26,95 \% \mathrm{Cl}$ : 0.06, 0.45)] and diastole [LVPWd, $(\beta$ $=0.18,95 \% \mathrm{Cl}: 0.06,0.30)]$, and thickened interventricular septal thickness in diastole [IVSd, $(\beta=0.16,95 \% \mathrm{Cl}: 0.05,0.28)]$. Boys with macrosomia showed a higher left ventricle mass index [LVMI, $(\beta=1.29,95 \% \mathrm{Cl}: 0.14,2.43)$ ], thickened LVPWs $(\beta=0.30$, 95\% Cl: 0.05, 0.56) and LVPWd ( $\beta=0.21,95 \% \mathrm{Cl}: 0.06,0.36)$, and thickened IVSd ( $\beta$ $=0.23,95 \% \mathrm{Cl}: 0.09,0.36)$. However, no significant association of structural changes 
was found in girls. Furthermore, an increased risk of LVH was found solely in macrosomic boys (OR $=2.79,95 \% \mathrm{Cl}: 1.17,6.63)$.

Conclusion: Children with macrosomia developed cardiovascular changes as early as 4 years of age. Macrosomia was associated with LV structural changes and higher LVH risk in pre-school-aged boys, while no association was found in girls.

Keywords: sex-disparity, birthweight, cardiovascular parameters, left ventricular hypertrophy, birth cohort study

\section{INTRODUCTION}

The impact of birthweight on cardiovascular health in later life has been acknowledged $(1,2)$. Previous studies found that children diagnosed with low birthweight (LBW) or macrosomia were prone to develop increased risk of cardiovascular disease in later life (3-5). This phenomenon was in accordance with the Barker hypothesis (6), which proposed that adverse prenatal exposure could alter metabolism and physiology in later life, and lead to increased risk of adult cardiovascular disease $(3,4,7)$.

Sex-disparity has been reported to exist in most noncommunicable diseases and certain infectious diseases (8-10). There was sufficient evidence indicating the sex difference in the incidence and prognosis of cardiovascular diseases (11-13). Previous studies demonstrated that sex-disparity in cardiovascular parameters could begin in early adolescence (14), but data at an early age was limited. As far as we know, there has been no report on the sex difference in the association between cardiovascular health and birthweight in pre-schoolaged children. Thus, the role of sex-disparity in the development of cardiovascular disease in early childhood remained unclear.

Based on the platform of Shanghai Birth Cohort (SBC), we aimed to examine the relationship between birthweight with the cardiovascular parameters, including blood pressure, and LV geometry and function, and further explore if sexdisparity existed during early childhood. The sex-specific risk of left ventricular hypertrophy (LVH) contributed by birthweight would also be investigated in this study.

\section{MATERIALS AND METHODS}

\section{Study Design and Participants}

The SBC was an ongoing prospective cohort study conducted in four large tertiary-level hospitals in Shanghai, China. The protocol of SBC has been previously described in detail (15, 16). Volunteer couples were recruited during preconception care or early pregnancy period from 2013 to 2016. Face-toface interviews were conducted by trained nurses to collect

\footnotetext{
Abbreviations: SBC, Shanghai Birth Cohort; LBW, low birthweight; NBW, normal birthweight; SBP, systolic blood pressure; DBP, diastolic blood pressure; BMI, body mass index; LV, left ventricle; LVH, left ventricle hypertrophy; LVMI, LV mass indexed to the height in $\mathrm{m}^{2.7}$; LVPWs, LV posterior wall thickness in systole; LVPWd, LV posterior wall thickness in diastole; LVDs, LV internal diameter in systole; LVDd, LV internal diameter in diastole; IVSs, interventricular septum thickness in systole; IVSd, interventricular septum thickness in diastole; RWT, relative wall thickness; LVEF, LV ejection fraction; GLS, global longitudinal strain; cIMT, carotid intima-media thickness.
}

information including parental age, race, educational levels, occupation, family income, alcohol use, and smoking status during pregnancy. The data related to newborns, regarding sex, gestational age, and anthropometric parameters, were recorded at birth by trained nurses. Information on maternal blood glucose and blood pressure levels was also collected from hospital records with the consent of patients. Those families still residing in Shanghai were invited to the prospective study. Detailed followup was provided for children at the age of 2 years and then at 4 years. Miscarriage, stillbirth, children with congenital heart defects, loss to follow-up in 4-year-olds, and without birthweight data were excluded. A total of 1,193 mother-offspring pairs were included in this study. Written informed consent was obtained from participants, and the study was approved by the Ethical Committee of Xinhua Hospital affiliated to Shanghai Jiao Tong University School of Medicine (no. XHEC-C-2013-001-2).

\section{Anthropometric Measurements}

A complete anthropometric evaluation was conducted for all children at birth, the age of 2 years, and 4 years according to the standardized protocol. Height was measured barefoot twice to the nearest $0.1 \mathrm{~cm}$. Weight was measured with light clothing and no shoes to the nearest $0.1 \mathrm{~kg}$. The average of two repeats was collected, and the third measurement was conducted if the two readings differed by $0.1 \mathrm{~kg}$ or $0.1 \mathrm{~cm}$. Body mass index (BMI) was calculated as weight $(\mathrm{kg}) /$ height ${ }^{2}\left(\mathrm{~m}^{2}\right)$.

The classification of birthweight was conducted according to the World Health Organization (17). Macrosomia was defined as a birthweight over 4,000 g. Normal birthweight (NBW) was defined as a birthweight between 2,500 and 4,000 g, and low birthweight (LBW) was $<2,500 \mathrm{~g}$.

\section{Measurement of Blood Pressure}

Blood pressure was measured from the left arm with appropriately sized cuff according to the guideline set by the American Academy of Pediatrics in 2017 (18), using the professional automatic BP monitor OMRAN HBP-1300 (Omron Healthcare, Guangzhou, CHINA) (19). Blood pressure was measured three times with a 5 min gap between replicates, after a comfortable rest of $5 \mathrm{~min}$. Measurements were done by trained coordinators in duplicate and averaged.

\section{Measurement of Cardiovascular Parameters in 4-Year-Olds}

Transthoracic echocardiography was performed for all children at the age of 4 years, by two experienced echocardiographers using an EPIQ 7C ultrasound system (Philips Healthcare, 
Andover, USA) equipped with S8-3 (8-3 MHz) and X5-1 (1$5 \mathrm{MHz}$ ) probes. Standard techniques were used to obtain Mmode, 2D, and Doppler measurements in accordance with the American Society of Echocardiography (20, 21). Quantitative measurements were obtained offline by well-trained investigators blinded to the data of subjects.

The dimensions of left ventricle (LV) were obtained from M-mode at parasternal short-axis view, including LV internal diameter indiastole and systole (LVDd and LVDs), interventricular septum thickness in diastole and systole (IVSd and IVSs), LV posterior wall thicknesses in diastole and systole (LVPWd and LVPWs), and left ventricular ejection fraction (LVEF) and fractional shortening (LVFS). Relative wall thickness (RWT) was calculated as (LVPWd + IVSd)/LVDd (22). The LV mass (LVM) was determined by the anatomically validated formula of Devereux (23) and the LVM index (LVMI) was calculated by dividing the LVM by the height 2.7 (24). The sexspecific 95th percentiles of LVMI derived from the cohort were used as the cutoff points since there was no recommended standard on LVMI for 4-year-old children in China (25). LVMI $=33.24 \mathrm{~g} / \mathrm{m}^{2.7}$ in girls and LVMI $=33.76 \mathrm{~g} / \mathrm{m}^{2.7}$ in boys represented the sex-specific 95th percentiles in the cohort. The LVH was defined as an LVMI more than the sex-specific 95th percentiles.

The ratio of early diastolic flow velocity (E) and late diastolic flow velocity (a) across mitral valve (E/a) was obtained by pulse wave Doppler in the apical 4-chamber view. We also got the left ventricle ejection time (ET), isovolumic contraction time (ICT), and isovolumic relaxation time (IRT) in the apical 5 -chamber view with the aid of pulse wave Doppler. The Tei index was calculated using the formula: Tei index $=$ $(\mathrm{ICT}+\mathrm{IRT}) / \mathrm{ET}$.

A 2-D speckle-tracking echocardiography (STE) analysis was performed using the commercial QLAB version 10.5 software (Philips Healthcare, Andover, USA) (26). To determine the global longitudinal strain (GLS), the software tracked the full wall region of interest automatically in the three apical views at the end of the diastole and allowed us to adjust the

TABLE 1 | Basic and cardiovascular characteristics of different birthweight groups.

\begin{tabular}{|c|c|c|c|c|}
\hline & $\begin{array}{c}\text { LBW } \\
3.86 \%(n=46)\end{array}$ & $\begin{array}{c}\text { NBW } \\
80.72 \%(n=963)\end{array}$ & $\begin{array}{c}\text { Macrosomia } \\
12.24 \%(n=184)\end{array}$ & $P$ \\
\hline Birthweight (g) & $2187.2 \pm 328.3$ & $3313.6 \pm 356.3$ & $4237.0 \pm 224.3$ & \\
\hline \multicolumn{5}{|l|}{ Gender } \\
\hline Boy, N (\%) & $23(50.0 \%)$ & $490(50.8 \%)$ & $103(56.0 \%)$ & 0.437 \\
\hline Girl, N (\%) & 23 (50.0\%) & $473(49.2 \%)$ & $81(44.0 \%)$ & 0.437 \\
\hline Weight at 4-yr (kg) & $16.93 \pm 2.09$ & $17.13 \pm 2.47$ & $18.18 \pm 3.18$ & $<0.001$ \\
\hline BMl at 4-yr $\left(\mathrm{kg} / \mathrm{m}^{2}\right)$ & $14.50 \pm 2.61$ & $14.75 \pm 2.16$ & $15.23 \pm 2.13$ & 0.013 \\
\hline $\mathrm{SBP}(\mathrm{mmHg})$ & $99.65 \pm 8.12$ & $97.87 \pm 7.74$ & $98.78 \pm 7.67$ & 0.148 \\
\hline $\mathrm{DBP}(\mathrm{mmHg})$ & $58.62 \pm 6.09$ & $57.41 \pm 6.09$ & $57.31 \pm 6.82$ & 0.444 \\
\hline \multicolumn{5}{|l|}{ LV structure at 4 years } \\
\hline LVDs (mm) & $22.13 \pm 1.84$ & $22.52 \pm 1.94$ & $22.95 \pm 2.10$ & 0.017 \\
\hline LVDd (mm) & $34.88 \pm 2.55$ & $35.40 \pm 2.51$ & $36.03 \pm 2.66$ & 0.006 \\
\hline IVSs (mm) & $6.37 \pm 0.88$ & $6.56 \pm 0.96$ & $6.67 \pm 1.14$ & 0.215 \\
\hline IVSd (mm) & $3.85 \pm 0.65$ & $3.79 \pm 0.57$ & $3.88 \pm 0.66$ & 0.156 \\
\hline RWT & $0.23 \pm 0.04$ & $0.24 \pm 0.04$ & $0.24 \pm 0.05$ & 0.244 \\
\hline \multicolumn{5}{|l|}{ LV function at 4 years } \\
\hline E/a & $1.77 \pm 0.34$ & $1.80 \pm 0.33$ & $1.79 \pm 0.38$ & 0.873 \\
\hline Tei index & $0.45 \pm 0.06$ & $0.42 \pm 0.06$ & $0.43 \pm 0.07$ & 0.337 \\
\hline LVEF (\%) & $60.08 \pm 4.98$ & $59.24 \pm 4.59$ & $59.16 \pm 4.72$ & 0.699 \\
\hline GLS (\%) & $23.33 \pm 2.28$ & $23.60 \pm 2.28$ & $23.59 \pm 2.51$ & 0.873 \\
\hline $\mathrm{clMT}(\mathrm{mm})$ & $41.26 \pm 4.69$ & $41.04 \pm 4.56$ & $40.92 \pm 4.59$ & 0.939 \\
\hline
\end{tabular}

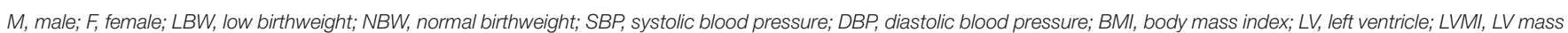

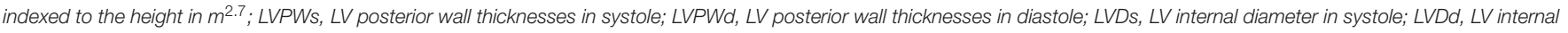

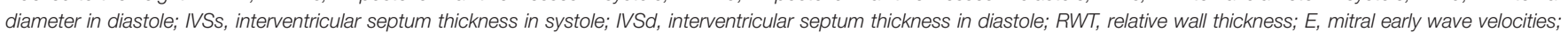

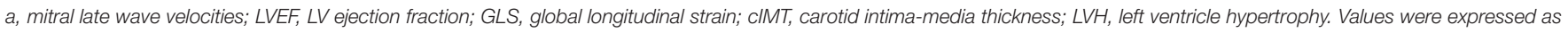
means \pm standard deviation or means with minimum and maximum. P-values were calculated using One-way ANOVA tests or Chi-square tests. 
tracking when necessary. In this case, a 17-segment model was used.

A 2-D ultrasound examination of both common carotid arteries was also conducted using the same high-resolution EPIQ 7C ultrasound equipped with linear array transducer (L15-7io; Philips Healthcare, Andover, USA) following a standardized protocol. Carotid intima-media thickness (cIMT) was determined as the distance between the lumen-intima interface and the media-adventitia interface. The measurement was performed twice on each side and the mean value was used for final analysis.

\section{Statistical Analysis}

Continuous variables were reported as means with standard deviation or means with minimum and maximum, and categorical variables were reported as percentages. One-way analysis of variance (one-way ANOVA) and Chi-square tests were used for comparison of basic characteristics and cardiovascular parameters among different birthweight groups in all children or different gender, and Bonferroni correction was used for multiple comparisons. Linear regression was used to assess all children and sex-specific associations between birthweight and cardiovascular parameters, using the NBW group as reference. Four models were conducted: model 1 was a crude model; model 2 was adjusted for maternal factors including maternal age, ethnicity (Han/Hui/Manchu/Mongolian/Zang/Uighur/Zhuang/Others/ unknown), educational level (never attended any school/primary school/junior high school/senior high school or technical school/junior college/undergraduate/postgraduate and above), alcohol intake (yes/no) and smoking status (yes/no) during pregnancy, gestational diabetes mellitus (yes/no), and

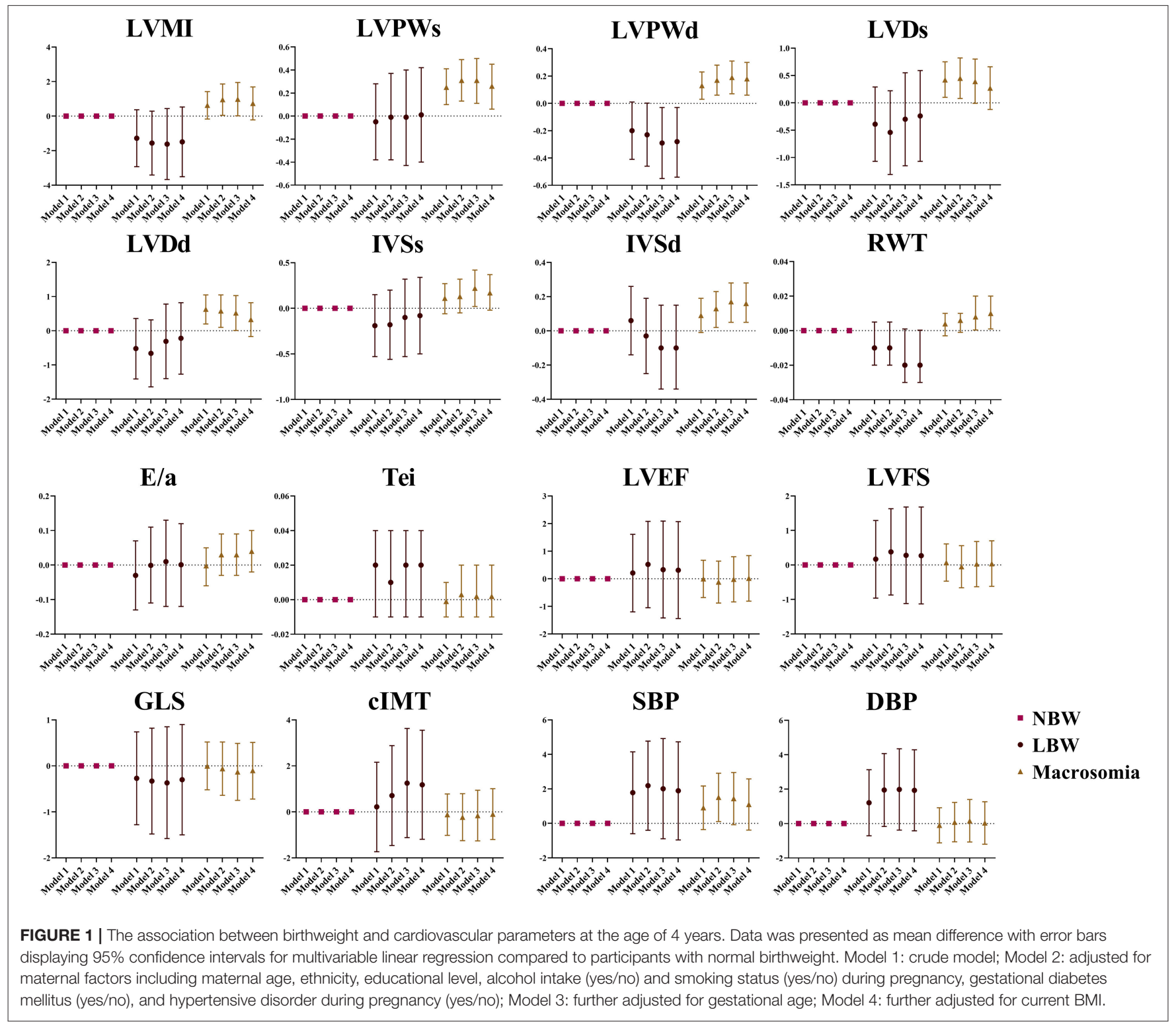


hypertensive disorder during pregnancy (yes/no); model 3 was further adjusted for gestational age; model 4 was further adjusted for current BMI. Maternal age, gestational age, and current BMI were treated as continuous variables in the regression analyses. Due to the low incidence of LVH in LBW group, we only explored the risk of LVH in macrosomia group with NBW group as reference. Logistic regression models were used to estimate the risk of left ventricular hypertrophy (LVH) in different birthweight subgroups, and maternal factors, gestational age, current BMI, and current blood pressure, including SBP and DBP were adjusted. All analyses were performed using Stata 14.0 software (StataCorp, College Station, TX, USA) and were two-sided, with $p<0.05$ considered as statistically significant.

\section{RESULTS}

\section{Association of Birthweight With Cardiovascular Outcome in 4-Year-Olds}

A total of 616 boys and 577 girls were included in this study. Among all the 1,193 children, 46 children were diagnosed with LBW (3.86\%), 963 children were diagnosed with NBW $(80.72 \%)$, and 184 children were diagnosed with macrosomia (12.24\%). Children showed LV structure changes at the age of 4 years, including LVPWs $(P=0.006)$, LVPWd $(P=$ $0.004)$, LVDs $(P=0.017)$, and $\operatorname{LVDd}(P=0.006)$, which were significantly different among three birthweight subgroups. However, the prevalence of $\mathrm{LVH}$ and other cardiovascular parameters, including blood pressure, LV structure parameters, and cIMT, showed no significant differences. The detailed information including demographic and anthropometric features of different birthweight subgroups is shown in Table 1.

The association between birthweight and cardiovascular parameters was further investigated among all the subjects. Positive association was found between children with macrosomia and LVPWs $(\beta=0.26,95 \%$ CI: $0.06,0.45)$, LVPWd ( $\beta=0.18,95 \%$ CI: $0.06,0.30)$, IVSd ( $\beta=0.16,95 \%$ CI: $0.05,0.28)$, and RWT ( $\beta=0.01,95 \%$ CI: $0.001,0.02)$. Negative association was found between LBW and LVPWd $(\beta=-0.28$, 95\% CI: $-0.54,-0.03)$. These results are shown in Figure 1.

\section{Sex-Disparity in the Association of Birthweight With Cardiovascular Outcome in 4-Year-Olds}

Among the children in this study, 23 (3.73\%) were diagnosed with LBW and $103(16.72 \%)$ were diagnosed with macrosomia among boys, while 23 (3.99\%) were diagnosed with LBW and 81 (14.04\%) were diagnosed with macrosomia among girls. Comparisons of cardiovascular parameters among different birthweight groups by gender are demonstrated in Table 2 . The LV structure variables such as LVMI $(P=0.007)$, LVPWs $(P=$ $0.008), \operatorname{LVPWd}(P=0.004), \operatorname{IVSd}(P=0.027)$, and the prevalence of LVH $(P=0.014)$ were significantly different among different birthweight groups in boys, which was not the case in girls. The blood pressure, LV function, and cIMT showed no significant difference in both boys and girls.
TABLE 2 | Sex-specific basic and cardiovascular characteristics of different birthweight groups.

\begin{tabular}{|c|c|c|c|c|}
\hline Cardiac parameters & LBW & NBW & Macrosomia & $P$-value \\
\hline \multicolumn{5}{|l|}{ Boy } \\
\hline SBP at 4 years $(\mathrm{mmHg})$ & $101.39 \pm 8.07$ & $99.12 \pm 7.92$ & $99.76 \pm 7.66$ & 0.348 \\
\hline DBP at 4 years $(\mathrm{mmHg})$ & $56.95 \pm 7.09$ & $57.15 \pm 6.29$ & $57.04 \pm 6.46$ & 0.981 \\
\hline LVMI $\left(\mathrm{g} / \mathrm{m}^{2.7}\right)$ & $24.75 \pm 3.50$ & $26.80 \pm 4.25$ & $28.06 \pm 5.36^{\# \S}$ & 0.007 \\
\hline LVPWs (mm) & $8.02 \pm 0.85$ & $7.95 \pm 0.94$ & $8.29 \pm 1.13^{\#}$ & 0.008 \\
\hline LVPWd (mm) & $3.95 \pm 0.52$ & $4.21 \pm 0.56$ & $4.39 \pm 0.73^{\# \S}$ & 0.004 \\
\hline LVDs (mm) & $22.79 \pm 2.06$ & $22.94 \pm 1.85$ & $23.43 \pm 2.06^{\#}$ & 0.068 \\
\hline LVDd (mm) & $36.16 \pm 2.64$ & $36.10 \pm 2.40$ & $36.76 \pm 2.39^{\#}$ & 0.052 \\
\hline IVSs (mm) & $6.61 \pm 0.90$ & $6.77 \pm 0.97$ & $6.83 \pm 1.04$ & 0.666 \\
\hline IVSd (mm) & $3.74 \pm 0.37$ & $3.80 \pm 0.53$ & $3.96 \pm 0.61^{\#}$ & 0.027 \\
\hline RWT & $0.22 \pm 0.03$ & $0.24 \pm 0.04$ & $0.24 \pm 0.05$ & 0.115 \\
\hline $\mathrm{E} / \mathrm{a}$ & $1.69 \pm 0.26$ & $1.79 \pm 0.33$ & $1.81 \pm 0.41$ & 0.352 \\
\hline Tei index & $0.44 \pm 0.06$ & $0.44 \pm 0.07$ & $0.43 \pm 0.07$ & 0.574 \\
\hline LVEF (\%) & $59.94 \pm 3.87$ & $58.87 \pm 4.51$ & $58.83 \pm 4.16$ & 0.731 \\
\hline GLS (\%) & $24.19 \pm 1.93$ & $23.44 \pm 2.28$ & $23.33 \pm 2.42$ & 0.520 \\
\hline clMT (mm) & $40.83 \pm 4.22$ & $41.33 \pm 4.68$ & $41.31 \pm 4.34$ & 0.946 \\
\hline LVH, N (\%) & $0(0.00 \%)$ & $32(6.53 \%)$ & $15(14.56 \%)^{\S}$ & 0.014 \\
\hline \multicolumn{5}{|l|}{ Girl } \\
\hline SBP at 4 years $(\mathrm{mmHg})$ & $97.83 \pm 7.95$ & $96.58 \pm 7.37$ & $97.32 \pm 7.56$ & 0.576 \\
\hline DBP at 4 years $(\mathrm{mmHg})$ & $60.38 \pm 7.57$ & $57.68 \pm 5.90$ & $57.63 \pm 7.32$ & 0.145 \\
\hline LVMI $\left(\mathrm{g} / \mathrm{m}^{2.7}\right)$ & $25.01 \pm 4.48$ & $25.51 \pm 4.98$ & $25.12 \pm 4.68$ & 0.770 \\
\hline LVPWs (mm) & $7.57 \pm 0.94$ & $7.70 \pm 0.85$ & $7.80 \pm 1.07$ & 0.535 \\
\hline LVPWd (mm) & $3.93 \pm 0.58$ & $4.06 \pm 0.57$ & $4.11 \pm 0.67$ & 0.496 \\
\hline LVDs (mm) & $21.59 \pm 1.47$ & $22.10 \pm 1.92$ & $22.28 \pm 1.99$ & 0.382 \\
\hline LVDd (mm) & $33.81 \pm 1.97$ & $34.68 \pm 2.41$ & $35.04 \pm 2.67$ & 0.148 \\
\hline IVSs (mm) & $6.18 \pm 0.84$ & $6.35 \pm 0.90$ & $6.46 \pm 1.23$ & 0.465 \\
\hline IVSd (mm) & $3.94 \pm 0.81$ & $3.78 \pm 0.61$ & $3.78 \pm 0.70$ & 0.573 \\
\hline RWT & $0.23 \pm 0.04$ & $0.24 \pm 0.04$ & $0.24 \pm 0.05$ & 0.952 \\
\hline$E / a$ & $1.81 \pm 0.36$ & $1.80 \pm 0.32$ & $1.78 \pm 0.34$ & 0.822 \\
\hline Tei index & $0.45 \pm 0.07$ & $0.43 \pm 0.06$ & $0.43 \pm 0.08$ & 0.273 \\
\hline LVEF (\%) & $60.24 \pm 6.20$ & $59.65 \pm 4.63$ & $59.69 \pm 5.53$ & 0.930 \\
\hline GLS (\%) & $22.38 \pm 2.33$ & $23.76 \pm 2.27$ & $24.02 \pm 2.64$ & 0.138 \\
\hline clMT (mm) & $41.61 \pm 5.20$ & $40.75 \pm 4.43$ & $40.42 \pm 4.91$ & 0.704 \\
\hline LVH, N (\%) & $1(3.40 \%)$ & $29(6.13 \%)$ & 4 (4.94\%) & 0.842 \\
\hline
\end{tabular}

LBW, low birthweight; NBW, normal birthweight; SBP, systolic blood pressure; DBP, diastolic blood pressure; BMI, body mass index; LV, left ventricle; LVMI, LV mass indexed to the height in $\mathrm{m}^{2.7}$; LVPWs, LV posterior wall thicknesses in systole; LVPWd, LV posterior wall thicknesses in diastole; LVDs, LV internal diameter in systole; LVDd, LV internal diameter in diastole; IVSs, interventricular septum thickness in systole; IVSd, interventricular septum thickness in diastole; RWT, relative wall thickness; E, mitral early wave velocities; a, mitral late wave velocities; LVEF, LV ejection fraction; GLS, global longitudinal strain; CIMT, carotid intima-media thickness; $L V H$, left ventricle hypertrophy. Values are means \pm standard deviation. P-values were calculated using One-way ANOVA tests or Chi-square tests, and Bonferroni correction was used for multiple comparisons. ${ }^{\#} P<0.05$ vs. NBW group.

$\S P<0.05$ vs. LBW group.

The sex-specific association between birthweight and cardiovascular outcome in 4-year-olds is represented in Figure 2. Positive association was found between boys with macrosomia and LVMI $(\beta=1.29,95 \%$ CI: $0.14,2.43)$, LVPWs $(\beta=0.30,95 \%$ CI: $0.05,0.56)$, LVPWd $(\beta=0.21,95 \%$ CI: $0.06,0.36)$, and IVSd 


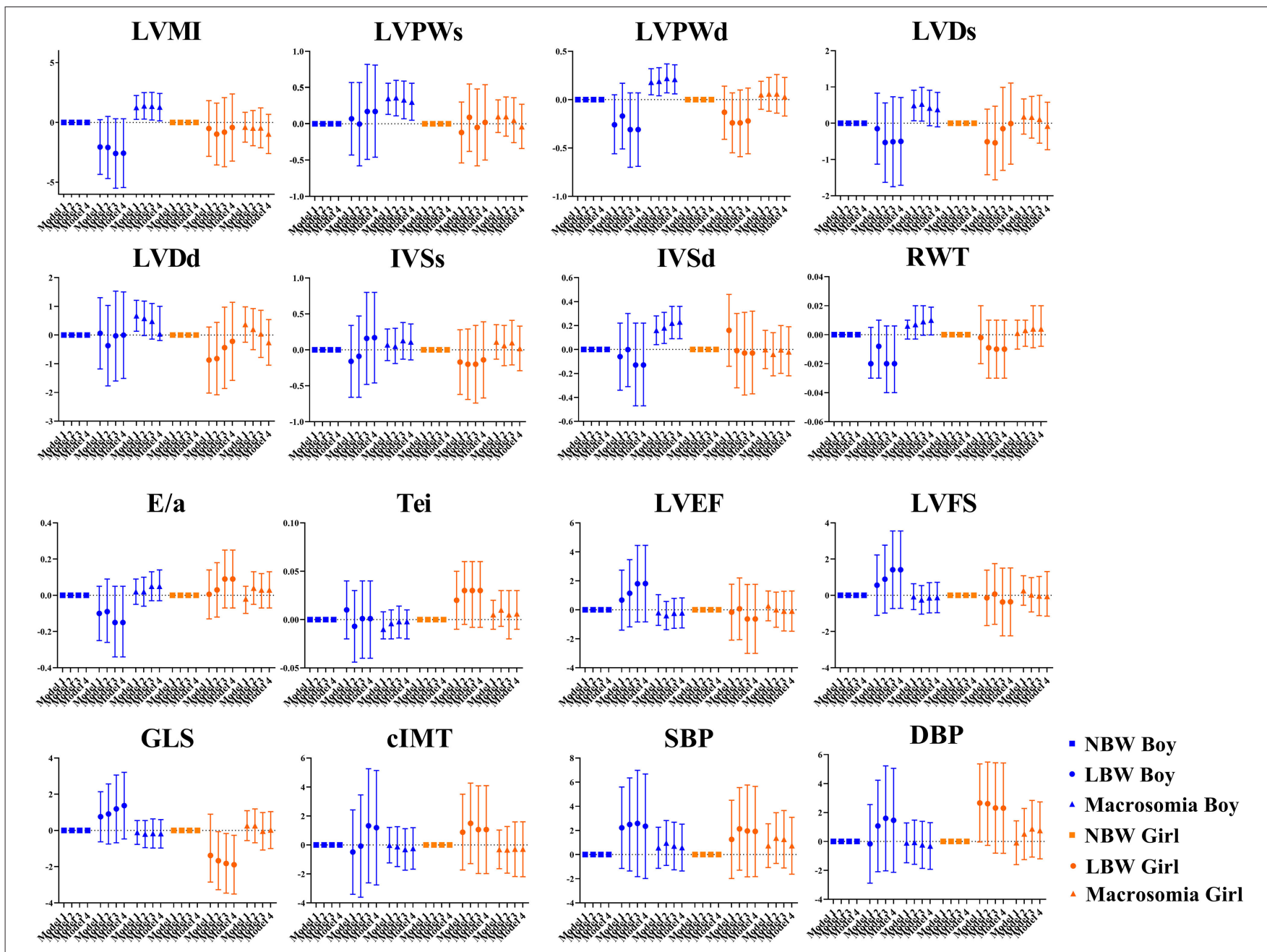

FIGURE 2 | Sex differences in the association between birthweight and cardiovascular parameters at the age of 4 years. Data was presented as mean difference with error bars displaying 95\% confidence intervals for multivariable linear regression compared to participants with normal birthweight. Model 1: crude model; Model 2: adjusted for maternal factors including maternal age, ethnicity, educational level, alcohol intake (yes/no) and smoking status (yes/no) during pregnancy, gestational diabetes mellitus (yes/no), and hypertensive disorder during pregnancy (yes/no); Model 3: further adjusted for gestational age; Model 4: further adjusted for current BMI.

$(\beta=0.23,95 \%$ CI: $0.09,0.36)$, while no statistical significance was found in blood pressure, cIMT, or LV function variables. Negative association was found between LBW girls and GLS $(\beta=-1.89,95 \% \mathrm{CI}:-3.51,-0.27)$. There was no obvious association found between 4 -year-old girls with macrosomia and cardiovascular outcome.

\section{Sex-Disparity in the Risk of Undesirable Cardiovascular Outcomes in 4-Year-Olds}

The risk of LVH in 4-year-old children with macrosomia is shown in Table 3. Children with macrosomia were conferred with a higher risk of LVH compared to NBW group with an odds ratio (OR) of 1.97 (95\% CI: 1.01, 3.85) with full adjustment. We further investigated the sex-specific relationship between macrosomia and risk of LVH. Boys with macrosomia were exposed to a higher risk of $\mathrm{LVH}$ with OR of 2.79 (95\% CI: 1.17, 6.63), indicating that macrosomia was an independent risk factor for LVH among pre-school-aged boys. Based on the results of logistic regression, higher birthweight seemed to have little effect on cardiovascular outcome in 4-year-old girls.

\section{DISCUSSION}

In this study, we demonstrated a significant association between birthweight and cardiovascular parameter changes in preschool-aged children. Furthermore, for the first time, we found sex-disparity in the association between birthweight and cardiovascular outcome, which developed at an early age of 4 years.

Screening of early cardiovascular changes in children was important as previous studies claimed that adverse LV structure and function changes were important factors influencing cardiac 
TABLE 3 | Odds ratios of LVH in the macrosomia group compared with the NBW group by gender.

\begin{tabular}{|c|c|c|c|c|}
\hline \multirow[t]{2}{*}{ Sex } & \multicolumn{2}{|c|}{ Crude model } & \multicolumn{2}{|c|}{ Adjusted model } \\
\hline & OR (95\% Cl) & $P$-value & OR (95\% Cl) & $P$-value \\
\hline \multicolumn{5}{|l|}{ Total } \\
\hline NBW & Reference & & Reference & \\
\hline Macrosomia & $1.69(0.98,2.93)$ & 0.059 & $1.97(1.01,3.85)$ & 0.046 \\
\hline \multicolumn{5}{|l|}{ Boys } \\
\hline NBW & Reference & & Reference & \\
\hline Macrosomia & $2.40(1.24,4.66)$ & 0.010 & $2.79(1.17,6.63)$ & 0.020 \\
\hline \multicolumn{5}{|l|}{ Girls } \\
\hline NBW & Reference & & Reference & \\
\hline Macrosomia & $0.82(0.28,2.40)$ & 0.712 & $1.17(0.29,4.72)$ & 0.824 \\
\hline \multicolumn{5}{|c|}{$\begin{array}{l}\text { LVH, left ventricle hypertrophy; NBW, normal birthweight; OR, odds ratio; Cl, } \\
\text { confidence interval. } \\
\text { Gestational age, current BMI, current SBP, current DBP and maternal factors including } \\
\text { maternal age, ethnicity, educational level, alcohol intake (yes/no) and smoking status } \\
\text { (yes/no) during pregnancy, gestational diabetes mellitus (yes/no), and hypertensive } \\
\text { disorder during pregnancy (yes/no) were further adjusted. Bold values in the table indicate } \\
\text { to statistically significant P-value. }\end{array}$} \\
\hline
\end{tabular}

remodeling $(27,28)$. Myocardial differences that developed with compensation at the expense of cardiac function in childhood could translate into undesirable cardiovascular outcomes in adulthood (29-31). Based on the comprehensive evaluation of cardiac structure and function, our results provided evidence for early detection of cardiac structural and functional changes in children with high risk at pre-school age. Some intervention studies have indicated that there was a reversibility in adverse cardiac adaption at early age, and we would discuss that phenomenon in our further longitudinal study.

In our study, sex-disparity was found to exist in the early adaptation of the cardiovascular system to adverse exposure during childhood. Among boys, the structural changes could develop earlier than functional changes, as cardiac structural changes and increased risk of LVH were observed at an early age of 4 years in children with macrosomia, whereas the cardiac function and peripheral vascular structure presented no obvious changes. However, no obvious structural changes were found in girls. Given the negative association found between LBW and GLS, we hypothesized that girls could develop functional changes earlier, rather than structural changes. Though a changes in GLS alone could not predict decline of cardiac function, it indicated an early-development of impaired contractile mechanics.

Furthermore, we revealed sex-disparity in the association between birthweight and cardiovascular outcome, indicating that there might be different determinants of cardiovascular outcome between boys and girls. The increased risk of LVH was found in boys with macrosomia, while there was no significant result found in girls. This finding was consistent with the results of some studies conducted in adults. Direct association between LVM at adolescence with birthweight $(32,33)$ and male sex (34) has been reported, which is in line with our results. Previous studies also found that LV structure was determined by somatic growth, and the current weight also played a role (35). Though there was no statistically significant result in girls, a slight upward trend of parameters related to LV geometry was still observed with increased birthweight. Based on the results of our study, we hypothesized that birthweight could be an important determinant in cardiovascular structure during early childhood for boys, and somatic growth and current body size could be more important for girls. This hypothesis needs further validation in a larger cohort study or animal models.

The possible mechanism of sex dimorphism in the increased risk of cardiovascular structural changes in macrosomic children is explained in the following lines. Genetic factors were considered first. The presence of sex chromosomes resulted in the difference between the bodies of males and females at the most fundamental genetic level (36-38). Indeed, some scholars has declared that every cell had its own sex (39). Followed by imprinting from parents, incomplete $\mathrm{X}$-inactivation, and epigenetic modification, the genes also influenced the development of cardiovascular organs (40). Besides, body fat distribution was another important factor. The difference in body fatness and adipose tissue distribution in different genders has been well documented (41). Males were more prone to the deposition of abdominal fat, especially around the abdominal internal organs. A previous study also reported a stronger increment in LVM in male mice when compared to female mice, on exposure to a high-fat diet (42). Furthermore, the sympathetic activation could also play a role. Many studies found that sympathetic activation was much stronger in men, and the sex-specific requirement for $\alpha 1$-adrenergic receptors had been previously reported (43). Although a body of evidence indicated that sex hormone had a large influence on the development of cardiovascular disease $(11,40,44)$, we thought that the effect of hormone in this study was little due to the slight difference in sex hormone in pre-school-aged children. Moreover, the social determinant difference such as physical activity should also be taken into consideration.

The investigation of our study was based on the prospective birth cohort study in China, with a detailed assessment of cardiovascular parameters during early childhood, which was seldom performed in previous epidemiology studies. Furthermore, for the first time, our study revealed that sex dimorphism developed in the association between macrosomia and increased risk of cardiovascular disease in early childhood. The limitations in our study should also be considered. Firstly, the risk of undesirable cardiovascular outcomes in children with LBW could not be fully explored due to fewer LBW children and low incidence of LVH among them. A larger sample size is expected for further investigation. Second, the influence of growth trajectories during early life was not considered for investigation in this study. As children with LBW/macrosomia usually presented a catch-up/catch-down growth (45-47), the role of the growth pattern in cardiovascular health among these children deserved more attention. Finally, information regarding physical activity was not included in this study. Physical activity was a risk factor for cardiovascular disease across the lifespan. The gender difference in adoption of health behaviors in early life has been illustrated (48), and it was observed that boys were encouraged for the greater pursuit of physical activity even from infancy. The gender difference and effect of physical activity need more consideration. 
In conclusion, early adaptation of the cardiovascular system with structural changes was found in children with LBW or macrosomia. For the first time, our study revealed the sex-disparity in the association between birthweight and cardiovascular parameters, where boys with macrosomia had heavier and thicker LV and were at a higher risk of LVH. Results of our work led to a better understanding of the sex-disparity in cardiovascular disease, providing evidence for more precise and effective interventions to reduce the risk of undesirable cardiovascular outcomes in children with risk factors of cardiovascular diseases.

\section{DATA AVAILABILITY STATEMENT}

The raw data supporting the conclusions of this article will be made available by the authors, without undue reservation.

\section{ETHICS STATEMENT}

The studies involving human participants were reviewed and approved by Ethical Committee of Xinhua Hospital Affiliated to Shanghai Jiao Tong University School of Medicine. Written informed consent to participate in this study was provided by the participants' legal guardian/next of kin.

\section{AUTHOR CONTRIBUTIONS}

KS, SC, and JW conceived and designed the study and collected data. HW and BD prepared an analytical plan, analyzed data, and drafted the initial manuscript. YW, YN, and $\mathrm{ZL}$ were involved in echocardiography examinations and data collection. FO collaborated in the revision and interpretation of the data and results. All authors reviewed and revised the manuscript, approved the final manuscript as submitted, and agreed to be accountable for all aspects of the work.

\section{FUNDING}

This work was supported by the National Key R\&D Program of China (grant nos: 2018YFC1002400 to 403), Collaborative Innovation Program of Shanghai Municipal Health Commission (grant no: 2020CXJQ01), and Hospital Funded Clinical Research, Clinical Research Unit, Xinhua Hospital Affiliated to Shanghai Jiao Tong University School of Medicine (grant no: 19XHCR06B).

\section{ACKNOWLEDGMENTS}

We gratefully acknowledge all the children and their parents for their participation and the research staff who have contributed to patient recruitment and data collection in the SBC.

\section{SUPPLEMENTARY MATERIAL}

The Supplementary Material for this article can be found online at: https://www.frontiersin.org/articles/10.3389/fnut.2021. 756512/full\#supplementary-material

\section{REFERENCES}

1. Mohseni R, Mohammed SH, Safabakhsh M, Mohseni F, Monfared ZS, Seyyedi J, et al. Birth weight and risk of cardiovascular disease incidence in adulthood: a dose-response meta-analysis. Curr Atheroscler Rep. (2020) 22:12. doi: 10.1007/s11883-020-0829-Z

2. Nordman H, Jääskeläinen J, Voutilainen R. Birth size as a determinant of cardiometabolic risk factors in children. Horm Res Paediatr. (2020) 93:14453. doi: 10.1159/000509932

3. Barker DJ, Winter PD, Osmond C, Margetts B, Simmonds SJ. Weight in infancy and death from ischaemic heart disease. Lancet. (1989) 2:57780. doi: 10.1016/s0140-6736(89)90710-1

4. Barker DJ, Osmond C, Forsén TJ, Kajantie E, Eriksson JG. Trajectories of growth among children who have coronary events as adults. $N$ Engl J Med. (2005) 353:1802-9. doi: 10.1056/NEJMoa044160

5. Boney CM, Verma A, Tucker R, Vohr BR. Metabolic syndrome in childhood: association with birth weight, maternal obesity, and gestational diabetes mellitus. Pediatrics. (2005) 115:e290-6. doi: 10.1542/peds.2004-1808

6. de Boo HA, Harding JE. The developmental origins of adult disease (Barker) hypothesis. Aust N Z J Obstet Gynaecol. (2006) 46:4-14. doi: 10.1111/j.1479-828X.2006.00506.x

7. Gram IT, Jacobsen BK, Straume B, Arnesen E, Løchen ML, Lund E. Early origin of coronary heart disease. Earlier published work supports the "Barker hypothesis”. BMJ. (1995) 310:1468. doi: 10.1136/bmj.310.6992.1468b

8. Shawihdi M, Dodd S, Kallis C, Dixon P, Grainger R, Bloom S, et al. Nationwide improvement in outcomes of emergency admission for ulcerative colitis in England, 2005-2013. Aliment Pharmacol Ther. (2019) 50:17692. doi: 10.1111/apt.15315

9. Pala L, Conforti F. Sex-based dimorphism in the SARS-CoV2 virulence. $J$ Intern Med. (2020) 288:477-8. doi: 10.1111/joim.13093

10. Clocchiatti A, Cora E, Zhang Y, Dotto GP. Sexual dimorphism in cancer. Nature reviews. Cancer. (2016) 16:330-9. doi: 10.1038/nrc.2016.30

11. Gerdts E, Regitz-Zagrosek V. Sex differences in cardiometabolic disorders. Nat Med. (2019) 25:1657-66. doi: 10.1038/s41591-019-0643-8

12. Miller V M. Universality of sex differences in cardiovascular outcomes: where do we go from here?. Eur Heart J. (2020) 41:1697-9. doi: 10.1093/eurheartj/ehaa310

13. Agarwala A, Michos ED, Samad Z, Ballantyne CM, Virani SS. The use of sexspecific factors in the assessment of women's cardiovascular risk. Circulation. (2020) 141:592-9. doi: 10.1161/CIRCULATIONAHA.119.043429

14. de Simone G, Devereux RB, Roman MJ, Ganau A, Chien S, Alderman $\mathrm{MH}$, et al. Gender differences in left ventricular anatomy, blood viscosity and volume regulatory hormones in normal adults. Am J Cardiol. (1991) 68:1704-8. doi: 10.1016/0002-9149(91)90333-g

15. Zhang J, Tian Y, Wang W, Ouyang F, Xu J, Yu X, et al. Cohort profile: the Shanghai Birth Cohort. Int J Epidemiol. (2019) 48:2121g. doi: 10.1093/ije/dyy277

16. Huo X, Huang R, Gan Y, Luo K, Aimuzi R, Nian M, et al. Perfluoroalkyl substances in early pregnancy and risk of hypertensive disorders of pregnancy: a prospective cohort study. Environ Int. (2020) 138:105656. doi: 10.1016/j.envint.2020.105656

17. Brämer GR. International statistical classification of diseases and related health problems. Tenth revision. World health statistics quarterly. Rapport Trimestriel de Stat Sanitaires Mondiales. (1988) 41:32-6.

18. Flynn JT, Kaelber DC, Baker-Smith CM, Blowey D, Carroll AE, Daniels SR. Clinical practice guideline for screening and management of high blood pressure in children and adolescents. Pediatrics. (2017) 140:e20171904. doi: 10.1542/peds.2017-1904

19. Meng L, Zhao D, Pan Y, Ding W, Wei Q, Li H, et al. Validation of Omron HBP-1300 professional blood pressure monitor based on 
auscultation in children and adults. BMC Cardiovasc Disord. (2016) 16:9. doi: 10.1186/s12872-015-0177-z

20. Lai WW, Geva T, Shirali GS, Frommelt PC, Humes RA, Brook MM, et al. Guidelines and standards for performance of a pediatric echocardiogram: a report from the task force of the pediatric council of the american society of echocardiography. J Am Soc Echocardiogr. (2006) 19:141330. doi: 10.1016/j.echo.2006.09.001

21. Lopez L, Colan SD, Frommelt PC, Ensing GJ, Kendall K, Younoszai AK, et al. Recommendations for quantification methods during the performance of a pediatric echocardiogram: a report from the pediatric measurements writing group of the american society of echocardiography pediatric and congenital heart disease council. J Am Soc Echocardiogr. (2010) 23:465577. doi: 10.1016/j.echo.2010.03.019

22. Biton Y, Goldenberg I, Kutyifa V, Baman JR, Solomon S, Moss AJ, et al. Relative wall thickness and the risk for ventricular tachyarrhythmias in patients with left ventricular dysfunction. J Am Coll Cardiol. (2016) 67:30312. doi: 10.1016/j.jacc.2015.10.076

23. Devereux RB, Alonso DR, Lutas EM, Gottlieb GJ, Campo E, Sachs I, et al. Echocardiographic assessment of left ventricular hypertrophy: comparison to necropsy findings. Am J Cardiol. (1986) 57:450-8. doi: 10.1016/0002-9149(86)90771-x

24. de Simone G, Daniels SR, Devereux RB, Meyer RA, Roman MJ, de Divitiis $\mathrm{O}$, et al. Left ventricular mass and body size in normotensive children and adults: assessment of allometric relations and impact of overweight. J Am Coll Cardiol. (1992) 20:1251-60. doi: 10.1016/0735-1097(92)90385-z

25. Sethna CB, Leisman DE. Left ventricular hypertrophy in children with hypertension: in search of a definition. Curr Hypertens Rep. (2016) 18:65. doi: 10.1007/s11906-016-0672-3

26. Voigt JU, Pedrizzetti G, Lysyansky P, Marwick TH, Houle H, Baumann $\mathrm{R}$, et al. Definitions for a common standard for $2 \mathrm{D}$ speckle tracking echocardiography: consensus document of the EACVI/ASE/Industry Task Force to standardize deformation imaging. J Am Soc Echocardiogr. (2015) 28:183-93. doi: 10.1016/j.echo.2014.11.003

27. Lai CC, Sun D, Cen R, Wang J, Li S, Fernandez-Alonso C, et al. Impact of long-term burden of excessive adiposity and elevated blood pressure from childhood on adulthood left ventricular remodeling patterns: the Bogalusa Heart Study. J Am Coll Cardiol. (2014) 64:1580-7. doi: 10.1016/j.jacc.2014.05.072

28. Van daele CM, De Meyer T, De Buyzere ML, Gillebert TC, Denil SL, Bekaert S, et al. Family history of cardiovascular disease and offspring echocardiographic left ventricular structure and function: the Asklepios Study. J Am Soc Echocardiogr. (2013) 26:1290-7.e2. doi: 10.1016/j.echo.2013.07.024

29. Gaasch WH, Zile MR. Left ventricular structural remodeling in health and disease: with special emphasis on volume, mass, and geometry. J Am Coll Cardiol. (2011) 58:1733-40. doi: 10.1016/j.jacc.2011.07.022

30. Cheng S, Vasan RS. Advances in the epidemiology of heart failure and left ventricular remodeling. Circulation. (2011) 124:e516-9. doi: 10.1161/CIRCULATIONAHA.111.070235

31. Thornburg K, Jonker S, O'Tierney P, Chattergoon N, Louey S, Faber J, et al. Regulation of the cardiomyocyte population in the developing heart. Prog Biophys Mol Biol. (2011) 106:289-99. doi: 10.1016/j.pbiomolbio.2010.11.010

32. de Jonge LL, van Osch-Gevers L, Willemsen SP, Steegers EA, Hofman A, Helbing WA, et al. Growth, obesity, and cardiac structures in early childhood: the Generation R Study. Hypertension. (2011) 57:93440. doi: 10.1161/HYPERTENSIONAHA.110.163303

33. Geelhoed JJ, Steegers EA, van Osch-Gevers L, Verburg BO, Hofman A, Witteman JC, et al. Cardiac structures track during the first 2 years of life and are associated with fetal growth and hemodynamics: the Generation R Study. Am Heart J. (2009) 158:71-7. doi: 10.1016/j.ahj.2009.04.018

34. Hietalampi H, Pahkala K, Jokinen E, Rönnemaa T, Viikari JS, Niinikoski $\mathrm{H}$, et al. Left ventricular mass and geometry in adolescence: early childhood determinants. Hypertension. (2012) 60:1266-72. doi: 10.1161/HYPERTENSIONAHA.112.194290

35. Janz KF, Dawson JD, Mahoney LT. Predicting heart growth during puberty: the muscatine study. Pediatrics. (2000) 105:E63. doi: 10.1542/peds.105.5.e63
36. Charchar FJ, Tomaszewski M, Lacka B, Zakrzewski J, ZukowskaSzczechowska E, Grzeszczak W, et al. Association of the human $\mathrm{Y}$ chromosome with cholesterol levels in the general population. Arterioscler Thromb Vasc Biol. (2004) 24:30812. doi: 10.1161/01.ATV.0000113291.39267.0a

37. Arnold AP. Rethinking sex determination of non-gonadal tissues. Curr Top Dev Biol. (2019) 134:289-315. doi: 10.1016/bs.ctdb.2019.01.003

38. Bloomer LD, Nelson CP, Denniff M, Christofidou P, Debiec R, Thompson $\mathrm{J}$, et al. Coronary artery disease predisposing haplogroup I of the $\mathrm{Y}$ chromosome, aggression and sex steroids-genetic association analysis. Atherosclerosis. (2014) 233:160-4. doi: 10.1016/j.atherosclerosis.2013.12.012

39. Institute of Medicine (US). Committee on understanding the biology of sex gender differences. In: Wizemann TM, Pardue ML, editors. Exploring the Biological Contributions to Human Health: Does Sex Matter?. Washington, DC: National Academies Press (US) (2001).

40. Regitz-Zagrosek V, Kararigas G. Mechanistic pathways of sex differences in cardiovascular disease. Physiol Rev. (2017) 97:1-37. doi: 10.1152/physrev.00021.2015

41. Mauvais-Jarvis F. Sex differences in metabolic homeostasis, diabetes, and obesity. Biol Sex Differ. (2015) 6:14. doi: 10.1186/s13293-015-0033-y

42. Böhm C, Benz V, Clemenz M, Sprang C, Höft B, Kintscher U, et al. Sexual dimorphism in obesity-mediated left ventricular hypertrophy. Am J Physiol Heart Circ Physiol. (2013) 305:H211-8. doi: 10.1152/ajpheart.00593.2012

43. Hart EC, Charkoudian N, Wallin BG, Curry TB, Eisenach JH, Joyner MJ. Sex differences in sympathetic neural-hemodynamic balance: implications for human blood pressure regulation. Hypertension. (2009) 53:5716. doi: 10.1161/HYPERTENSIONAHA.108.126391

44. Donaldson C, Eder S, Baker C, Aronovitz MJ, Weiss AD, Hall-Porter M, et al. Estrogen attenuates left ventricular and cardiomyocyte hypertrophy by an estrogen receptor-dependent pathway that increases calcineurin degradation. Circ Res. (2009) 104:265-75. doi: 10.1161/CIRCRESAHA.108. 190397

45. Lei X, Zhao D, Huang L, Luo Z, Zhang J, Yu X, et al. Childhood health outcomes in term, large-for-gestational-age babies with different postnatal growth patterns. Am J Epidemiol. (2018) 187:507-14. doi: 10.1093/aje/ kwx271

46. Ong KK. Catch-up growth in small for gestational age babies: good or bad? Curr Opin Endocrinol Diabetes Obes. (2007) 14:30-4. doi: 10.1097/MED.0b013e328013da6c

47. Renom Espineira A, Fernandes-Rosa FL, Bueno AC, de Souza RM, Moreira AC, de Castro $\mathrm{M}$, et al. Postnatal growth and cardiometabolic profile in young adults born large for gestational age. Clin Endocrinol. (2011) 75:33541. doi: 10.1111/j.1365-2265.2011.04054.x

48. O'Neil A, Scovelle AJ, Milner AJ, Kavanagh A. Gender/Sex as a social determinant of cardiovascular risk. Circulation. (2018) 137:854-64. doi: 10.1161/CIRCULATIONAHA.117.028595

Conflict of Interest: The authors declare that the research was conducted in the absence of any commercial or financial relationships that could be construed as a potential conflict of interest.

Publisher's Note: All claims expressed in this article are solely those of the authors and do not necessarily represent those of their affiliated organizations, or those of the publisher, the editors and the reviewers. Any product that may be evaluated in this article, or claim that may be made by its manufacturer, is not guaranteed or endorsed by the publisher.

Copyright $\odot 2021$ Wang, Du, Wu, Li, Niu, Ouyang, Wang, Chen and Sun. This is an open-access article distributed under the terms of the Creative Commons Attribution License (CC BY). The use, distribution or reproduction in other forums is permitted, provided the original author(s) and the copyright owner(s) are credited and that the original publication in this journal is cited, in accordance with accepted academic practice. No use, distribution or reproduction is permitted which does not comply with these terms. 